\section{Asperger Syndrome}

Edited by Ami Klin, Fred R. Volkmar \& Sara S. Sparrow. New York: Guilford Press. 2000. 489 pp. $€ 32.50$ (hb). ISBN I 572305347



Call me sad, but when I was asked to review Asperger Syndrome my heart skipped a beat. The reasons: increasing numbers of people are being referred to mental health services for advice on 'Asperger's', yet little is known about the disorder; the editors of this book are pre-eminent in their field; and the people contributing chapters include outstanding researchers and affected individuals. So, what do we know already, and what was I hoping to learn?

We know that autistic disorder (comprising the genetically related subtypes of classic autism, high-functioning autism and Asperger syndrome) is a developmental neuropsychiatric condition and that it is associated with a significant increase in social exclusion and mental health problems. Core symptoms include impaired development of reciprocal social skills and communication, ritualistic/compulsive behaviour. Individuals with classical autism also have delayed language development and most have learning disability. However, around $25 \%$ are classified as highfunctioning, because they have normal or superior general intellectual ability, in spite of having a history of early language delay. Individuals with Asperger syndrome have no history of language delay and have normal or superior intellectual abilities, but also show the characteristic impairments in reciprocal social interaction (Wing,
1997; Gillberg, 1998). Thus, in both highfunctioning autism and Asperger syndrome there is a significant dissociation between cognitive and social skills.

There are also many things we do not know about Asperger syndrome. These include its genetic and neurobiological determinants, comorbidity with other mental health disorders, response to treatment, long-term outcome and the health and social costs to the person and their family.

Does this book help illuminate these issues and would I recommend it to researchers, clinicians and people affected by Asperger syndrome? My answers are "Well, sort of" and (respectively) "Yes", "No" and "No". The chapters on historical aspects, differential diagnosis, neuropsychology, brain imaging, language disorders and non-verbal learning disabilities are illuminating, and the contributions by affected people are valuable. However, there is little new information on issues of assessment, treatment or outcome. Also, some chapters seemed to be based mainly on work in classical or high-functioning autism. This is almost inevitable because our knowledge about Asperger syndrome is relatively limited. None the less, I could not help feeling that the book, while well intentioned and responding to our demand for more information, is slightly premature, given the current level of knowledge.

Gillberg, C. (1998) Asperger syndrome and highfunctioning autism. British Journal of Psychiatry, I72, 200-209.

Wing, L. (1997) The autistic spectrum. Lancet, 350, 1761-1766

Declan G. M. Murphy Professor of Psychiatry and Brain Maturation, Division of Psychological Medicine, Institute of Psychiatry, De Crespigny Park, Denmark Hill, London SE5 8AF, UK, and Honorary Consultant Psychiatrist, South London and Maudsley NHS Trust

\section{Behavior and Mood Disorders in Focal Brain Lesions}

Edited by Julien Bogousslavsky \& Jeffrey L.Cummings. Cambridge: Cambridge University Press. 2000.554 pp. $€ 49.95$ (pb). ISBN 052177482

The structure of this volume is theoretically based on a trichotomy, first developed by Albert, between instrumental, fundamental and executive cerebral functions. The first are essentially related to communication and are represented by cortical activities; fundamental refers to learning, information processing and mood; and executive functions are those related to abstraction, sequencing and attentional activities. The last two are predominantly subcortically and frontally driven, respectively. Abnormalities of the cerebral structures underlying these domains are represented by signature syndromes, which form the main text of the book.

After a brief review of relevant neuroanatomy, there follows a succession of chapters dominated either by a syndrome (e.g. depression and lesion location in stroke) or by anatomy (e.g. thalamic behaviour syndromes). The chapters are variable in their comprehensibility and length, and there is much repetition between chapters, which considerably lengthens the book and reduces its reader-friendliness. Furthermore, the trichotomy introduced in the discussion is somehow lost sight of.

There is a disappointing adherence to the value of using DSM-IV in behavioural neurology, and the subtlety of mental-state changes specifically related to neurological damage is not well developed. An obvious example is in the field of epilepsy. In the chapter on the evaluation of behaviour, in which behavioural rating scales are discussed, the Bear-Fedio inventory (introduced to evaluate temporal lobe syndromes) is not listed. In the chapter on temporal lobe syndromes, one of the most specific of all neurobehavioural abnormalities, the postictal psychosis of temporal lobe epilepsy is not even mentioned. Of the five references given to support the contention that there is no link between temporal lobe epilepsy and psychosis, one is a review article and the other four support the proposition that there is a link.

These are perhaps quibbles about a book that undoubtedly contains some excellent review chapters, all well referenced, and of considerable value for those references alone. There is a wealth of important behavioural neurology within it and there is no comparable text that covers this important topic.

The book is dominated by French and American authors, and it is significant to have chapters by French behavioural neurologists. Since the time of Charcot there have been many contributions to neuropsychiatry from France, but they are often not well represented in contemporary texts. How- 
ever, there is still a need for a thoughtful, comprehensive, single-authored text to bring together our knowledge of the behavioural consequences of focal brain lesions, set in a connectionist context and not dominated by DSM-IV.

R. Trimble Institute of Neurology, Queen Square, London WCIN 3BG, UK

\section{Race, Culture and Ethnicity in Secure Psychiatric Practice: Working with Difference}

Edited by Charles Kay \& Tony Lingiah. London: Jessica Kingsley. 2000. 284 pp. €47.50 (hb); £I8.95 (pb). ISBN I 853026956 (hb); ISBN I 853026954 (pb)

A White middle-class Scot must approach issues of race and culture in forensic practice with uncertainty. This book aims to help clinicians like me and deserves a place in the libraries of forensic units. It is, however, fragmented and incomplete in its coverage, let down by its editing and presentation.

There are interesting contributions from users and reflections on practice described by professionals. Case vignettes bring issues to life. For me with two Muslim patients currently on my ward, the description of Islam and the experience of the visiting Imam to Broadmoor have been extremely helpful. An account of a social worker's experience alongside Asian women with mental health problems in the community is similarly illuminating, with valuable explanations of the importance of biradari (kinship) and izzat (pride) in how mental health difficulties are experienced.

Many forensic practitioners will read the title and immediately think of the debate surrounding the relationship between African-Caribbean origin, schizophrenia and offending activity. It is a daunting task to present a balanced review of this literature and the contributions here that attempt to address this topic are disappointing. The key chapter examining this relationship is a revised, updated and expanded version of a summary report first commissioned and published by the Mental Health Foundation in 1995. Long lists of publications do not encourage understanding of the important arguments involved, and more use could have been made of tables, summarising key findings. Several of the arguments and references are revisited in other contributions, but the authors do not appear to have had sight of these and cross-referencing is lacking.

Although the book has some effective illustrations that highlight cultural differences in, for example, art work, many of the graphs are poorly reproduced and difficult to interpret. References are confusingly cited and are sometimes inaccurate. Despite these reservations, I would encourage forensic practitioners to read this book. It contains some outstanding individual contributions, answers to specific cultural questions and provides resources to help address racial issues in secure services, in the form of policy statements and audit protocols from Broadmoor Hospital.

John Crichton Consultant Forensic Psychiatrist, The Orchard Clinic, Royal Edinburgh Hospital, Morningside Terrace, Edinburgh EHIO 5HF, UK

\section{The International Handbook of Suicide and Attempted Suicide}

Edited by Keith Hawton and Kees van Heeringen. Chichester: John Wiley \& Sons. 2000.755 pp. $€ 75.00$ (hb).

ISBN 0 47I 983675

No self-respecting worker in deliberate self-harm and suicide prevention, either clinical or research, can afford to be without access to this comprehensive handbook - possession (or at least, a copy in one's local library) and regular use, may well become a marker of serious involvement in the subject! Every university department of psychiatry, and every major hospital with a medical accident and emergency department striving to carry out their work to a high standard will need to have this accessible and well-thumbed.

This is the most comprehensive, up-todate, informative and well-written source of information on suicide and suicidal behaviour. It integrates knowledge in terms of the interaction between the individual and the environment with relation to all aspects of suicidal behaviour. There are four parts. The first, on understanding suicidal behaviour, deals in detail with background factors for suicide and deliberate self-harm: epidemiology worldwide, psychology, ethology, sociology and genetics. Psychiatric aspects of suicidal behaviour are considered in terms of depression, anxiety disorders, schizophrenia, substance misuse and personality disorders. An excellent chapter integrates these aetiological factors and delineates the pathways to suicide, how these different factors combine.

The second part considers different populations (children, adolescents and the elderly) and their association with suicide. It also looks at associations with sexuality and the reproductive cycle, employment and the labour market, the impact of suicide on relatives and friends, ethical and legal issues, repeated suicidal attempts, physically and psychiatrically ill populations, and the link between suicide and violence.

The third part covers pharmacological and psychological approaches for the treatment of suicidal behaviour. In particular, it considers people who attempt suicide in adolescence and their treatment, prevention in the elderly and management in the general hospital. There is a chapter on multi-disciplinary approaches to management. In addition to the methods of treatment described, the evaluation of outcome data is also extremely valuable for planning services.

The final section is concerned with the prevention of suicide and attempted suicide. It looks at the prediction of suicide and also at strategies for the general population, including a chapter specifically

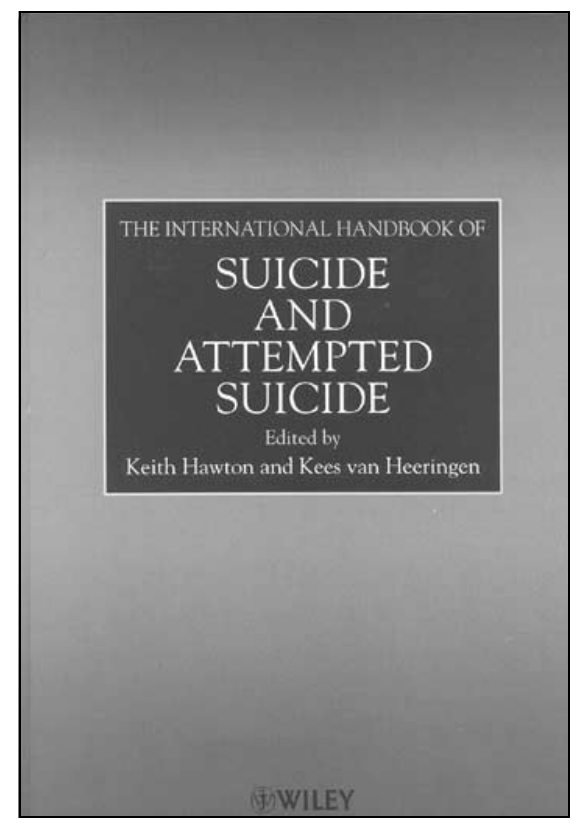

